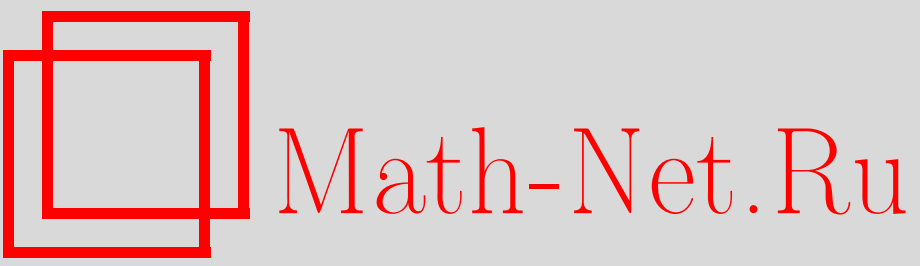

Н. Е. Ратанов, Случайные блуждания частицы в неоднородной одномерной среде с отражением и поглощением, ТМФ, 1997, том 112, номер 1, 81-91

DOI: https://doi.org/10.4213/tmf1028

Использование Общероссийского математического портала Math-Net.Ru подразумевает, что вы прочитали и согласны с пользовательским соглашением

http://www.mathnet.ru/rus/agreement

Параметры загрузки:

IP : 54.197 .130 .99

26 апреля 2023 г., 16:42:09 
ТЕОРЕТИЧЕСКАЯ

И МАТЕМАТИЧЕСКАЯ

ФИЗИКА

Том 112, № 1

июль, 1997

\author{
Н.Е. Ратанов*
}

\title{
СЛУЧАЙНЫЕ БЛУЖДАНИЯ ЧАСТИЦЫ В НЕОДНОРОДНОЙ ОДНОМЕРНОЙ СРЕДЕ С ОТРАЖЕНИЕМ И ПОГЛОЩЕНИЕМ
}

Рассматривается процесс движения в одномерной неоднородной среде частицы, претерпевающей в пуассоновские моменты времени изменения направления скорости. Для такого случайного процесса выведены обратное и прямое уравнения Колмогорова. Получены формулы для распределений как исходного процесса, так и аналогичных процессов в присутствии отражаюших и поглощающих барьеров.

\section{1. ВВЕДЕНИЕ}

Пусть $N(t), t \geq 0,-$ стационарный пуассоновский процесс с параметром $\mu>0$, $\xi$ - случайная величина, не зависимая от $N(t)$ и принимающая значения \pm 1 с вероятностью $1 / 2$. Пусть $c(x), x \in(-\infty, \infty),-$ положительная непрерывная функция. В работе рассматриваются процессы, задаваемые уравнением

$$
X(x, t)=x+\xi \int_{0}^{t}(-1)^{N(s)} c(X(x, s)) d s, \quad x \in(-\infty, \infty), \quad t>0,
$$

а также аналогичные процессы в присутствии отражаюших и поглошаюших барьеров. Процесс (1.1) описывает блуждание в одномерной неоднородной среде частицы, претерпевающей в пуассоновские моменты времени изменения направления движения. Такого рода модели естественно возникают в некоторых задачах физики, в особенности в тех задачах диффузии, для которых является сушественной конечность скорости распространения возмушения. Изучению процессов вида (1.1) посвящено большое количество математических и физических работ [1-5].

Однако все известные результаты в этой области относятся к блужданиям в однородных средах, т.е. к случаю $c(x) \equiv$ const. Одной из целей статьи является распространение результатов работы Орсингера [2] на неоднородный $(c(x) \not \equiv$ const) случай. Следует отметить, что для процесса (1.1) в отличие от однородного случая многие известные результаты выглядят более естественно.

\footnotetext{
* Челябинский государственный университет, Челябинск, Россия
} 
Основные результаты данной статьи состоят в явном вычислении распределения процесса (1.1) как в случае “свободного" движения, так и в присутствии отражающих и поглощающих экранов. Такое вычисление основьвается на том обстоятельстве, что распределение процесса (1.1) является решением телеграфного уравнения

$$
\frac{\partial^{2} u(x, t)}{\partial t^{2}}+2 \mu \frac{\partial u(x, t)}{\partial t}=c(x) \frac{\partial}{\partial x} c(x) \frac{\partial}{\partial x} u(x, t), \quad x \in(-\infty, \infty), \quad t>0 .
$$

Это утверждение, сформулированное впервые Голдстейном [6] и Кацем [7], было впоследствии доказано для случая $c(x) \equiv$ const во многих работах (см., например, $[8,9])$. Доказательства в [8] и [9], основанные на принципиально различных идеях, приводят, однако, к одному и тому же уравнению.

В разделах 2 и 3 данной статьи показано, что для блуждания в неоднородной среде (1.1) подход Орсингера [8] ведет к уравнению, аналогичному прямому уравнению Колмогорова, тогда как идея Кабанова [9] приводит к обратному уравнению Колмогорова. В четвертом разделе обсуждаются изменения, которые вносит присутствие барьеров. Раздел 5 содержит явные формулы для решений уравнения (1.2).

\section{2. ВЫВОД ОБРАТНОГО УРАВНЕНИЯ КОЛМОГОРОВА}

В этом разделе мы кратко повторяем аргументы Кабанова [9] в случае переменной скорости движения $c(x)$.

Пусть $c \in C^{1}(-\infty, \infty), c(x)>0 \forall x \in(-\infty, \infty)$. Обозначим через $X^{ \pm}$решения уравнений

$$
X^{+}(x, t)=x+\int_{0}^{t}(-1)^{N(s)} c\left(X^{+}(x, s)\right) d s
$$

и

$$
X^{-}(x, t)=x-\int_{0}^{t}(-1)^{N(s)} c\left(X^{-}(x, s)\right) d s,
$$

где $X^{+}$и $X^{-}-$это блуждания частишы с начальными скоростями $c(x)$ и $-c(x)$, соответственно.

Теорема 2.1. Пусть $\varphi=\varphi(x), \quad x \in(-\infty, \infty),-$ некоторая гладкая функция. Функиии $u^{ \pm}=u^{ \pm}(x, t)=E \varphi\left(X^{ \pm}(x, t)\right), \quad x \in(-\infty, \infty), \quad t>0$, удовлетворяют телеграфному уравнению

$$
\frac{\partial^{2} u^{ \pm}(x, t)}{\partial t^{2}}+2 \mu \frac{\partial u^{ \pm}(x, t)}{\partial t}=c(x) \frac{\partial}{\partial x} c(x) \frac{\partial}{\partial x} u^{ \pm}(x, t), \quad x \in(-\infty, \infty), \quad t>0
$$

и начальным мсловиям

$$
\left.u^{ \pm}\right|_{t=0}=\varphi(x),\left.\quad u_{t}^{ \pm}\right|_{t=0}= \pm c(x) \varphi^{\prime}(x) .
$$

Здесь и всюду ниже $E(\cdot)$ обозначает математическое ожидание.

Докажем сначала следующую лемму. 
Лемма 2.1. Пусть $s(t)=(-1)^{N(t)}$, где $N(t)$ - процесс Пуассона с показателем $\mu$. Пусть $X(t)$ - неупрежсдающий по отношению $\kappa N(t)$ процесс, $f$ - измеримая функиия такая, что $f(X(t))$ - случайньй процесс. Тогда

$$
E f(X(t)) d s(t)=-2 \mu E f(X(t)) s(t) d t .
$$

ДокАЗАтЕльСтво леммы (ср. [9]). Рассмотрим $m(t)=s(t) e^{2 \mu t}$. Используя это обозначение, нетрудно увидеть, что процесс $s(t)$ удовлетворяет уравнению $d s(t)=$ $-2 \mu m(t) e^{-2 \mu t} d t+e^{-2 \mu t} d m(t)$. Следовательно,

$$
E f(X(t)) d s(t)=-2 \mu E f(X(t)) m(t) e^{-2 \mu t} d t+e^{-2 \mu t} E f(X(t)) d m(t) .
$$

Последнее слагаемое равно нулю, поскольку $m(t)$ является мартингалом. Лемма доказана.

ДОКАЗАТЕЛЬСТВО теоремы 2.1 состоит в прямом вычислении с использованием леммы 2.1. Из (2.1) и (2.2) вытекает, что

$$
\frac{\partial X^{ \pm}}{\partial t}(x, t)= \pm(-1)^{N(t)} c\left(X^{ \pm}(x, t)\right)
$$

$$
\int_{x}^{X^{ \pm}} \frac{d y}{c(y)}= \pm \int_{0}^{t}(-1)^{N(s)} d s .
$$

Дифференцируя (2.7) по $x$, имеем

$$
\frac{\partial}{\partial x} X^{ \pm}(x, t)=\frac{c\left(X^{ \pm}(x, t)\right)}{c(x)} .
$$

Имея в виду (2.6), можно вычислить производные $u^{ \pm}$по $t$ :

$$
\frac{\partial u^{ \pm}}{\partial t}= \pm E \varphi^{\prime}\left(X^{ \pm}\right) c\left(X^{ \pm}\right) s(t)
$$

Отсюда формально получаем (см. аккуратное доказательство в [9])

$$
\frac{\partial^{2} u^{ \pm}}{\partial t^{2}}=E \varphi^{\prime \prime}\left(X^{ \pm}\right) c\left(X^{ \pm}\right)^{2}+E \varphi^{\prime}\left(X^{ \pm}\right) c^{\prime}\left(X^{ \pm}\right) c\left(X^{ \pm}\right) \pm E \varphi^{\prime}\left(X^{ \pm}\right) c\left(X^{ \pm}\right) \dot{s}(t) .
$$

По лемме 2.1 последнее слагаемое равно

$$
\mp 2 \mu E \varphi^{\prime}\left(X^{ \pm}\right) c\left(X^{ \pm}\right) s(t)=-2 \mu \frac{\partial u^{ \pm}}{\partial t} .
$$

Для дифференцирования по $x$ используем (2.8):

$$
\begin{gathered}
c(x) \frac{\partial u^{ \pm}}{\partial x}=E \varphi^{\prime}\left(X^{ \pm}\right) c\left(X^{ \pm}\right) \\
c(x) \frac{\partial}{\partial x} c(x) \frac{\partial u^{ \pm}}{\partial x}=E\left(\varphi^{\prime \prime}\left(X^{ \pm}\right) c\left(X^{ \pm}\right)^{2}+\varphi^{\prime}\left(X^{ \pm}\right) c^{\prime}\left(X^{ \pm}\right) c\left(X^{ \pm}\right)\right) .
\end{gathered}
$$

Таким образом, $u^{ \pm}(x, t)$ удовлетворяет (2.3). Проверка выполнения начальных условий (2.4) также не составляет труда.

Из доказанного вытекает следуюшая теорема. 
ТеОрема 2.2 (ср. с [9]). Решение задачи Коши для уравнения (2.3) с начальными условиями

$$
\left.u\right|_{t=0}=\varphi(x),\left.\quad u_{t}\right|_{t=0}=0
$$

имеет вид

$$
u(x, t)=\frac{1}{2} E\left[\varphi\left(X^{+}(x, t)\right)+\varphi\left(X^{-}(x, t)\right)\right]
$$

ЗАмЕчАниЕ 2.1. Уравнение (2.3) естественно называть обратным уравнением Колмогорова для процесса $X(x, t)$. В разделе 5 приведены формулы, разрешающие задачи $(2.3),(2.4)$ и $(2.3),(2.9)$. Отметим, что для случая постоянных коэффициентов эти формулы хорошо известны $[2,10]$.

Определим $p(x, y, t)$ и $p^{ \pm}(x, y, t)$ - плотности распределения процессов $X(x, t)$ и $X^{ \pm}(x, t)$ соотношениями

$$
\begin{gathered}
E \varphi(X(x, t))=\langle p(x, \cdot, t), \varphi\rangle, \\
E \varphi\left(X^{ \pm}(x, t)\right)=\left\langle p^{ \pm}(x, \cdot, t), \varphi\right\rangle, \quad x \in(-\infty, \infty), \quad t>0, \\
\varphi \in C_{0}^{\infty}\left(\mathbb{R}^{1}\right) .
\end{gathered}
$$

Здесь и всюду ниже $\langle\cdot, \cdot\rangle$ обозначает действие обобшенной функции на основную.

СлеДСТВИЕ 2.1. Функиии $p(x, y, t), \quad p^{ \pm}(x, y, t)$ удовлетворяют телеграфному уравнению (2.3) и следующим начальным условиям:

$$
\begin{aligned}
\left.p\right|_{t=0} & =\delta(x-y), & \left.p_{t}\right|_{t=0} & =0, \\
\left.p^{ \pm}\right|_{t=0} & =\delta(x-y), & \left.p_{t}^{ \pm}\right|_{t=0} & = \pm c(x) \delta^{\prime}(x-y) .
\end{aligned}
$$

Здесь $\delta$ обозначает $\delta$-функцию Дирака.

ЗАмЕчАниЕ 2.2. Ясно, что условие гладкости коэффициентов $c \in C^{1}(-\infty, \infty)$ можно заменить условием кусочной гладкости. Именно, достаточно считать, что $c \in$ $C(-\infty, \infty)$, а производная $c^{\prime}(x)$ сушествует всюду за исключением дискретного набора точек.

\section{3. ВЫВОД ПРЯМОГО УРАВНЕНИЯ КОЛМОГОРОВА}

ТЕОРема 3.1. Функиия $p(x, y, t)$, определенная в $(2.11)$, является решением следующей задачи Коши:

$$
\begin{gathered}
\frac{\partial^{2}}{\partial t^{2}} p(x, y, t)+2 \mu \frac{\partial}{\partial t} p(x, y, t)=\frac{\partial}{\partial y} c(y) \frac{\partial}{\partial y} c(y) p(x, y, t), \\
\left.p\right|_{t=0}=\delta(x-y),\left.\quad \frac{\partial p}{\partial t}\right|_{t=0}=0 .
\end{gathered}
$$


ДокАЗАТЕльСтво. Следуя Орсингеру [1], введем функции распределения

$$
\begin{aligned}
& F(x, y, t)=P\{X(x, t)<y, \quad V(x, t)>0\}, \\
& B(x, y, t)=P\{X(x, t)<y, \quad V(x, t)<0\},
\end{aligned}
$$

где $V(x, t)$ обозначает текущую скорость: $V(x, t)=\xi(-1)^{N(t)} c(X(x, t))$. Обозначим через $f(x, y, t)$ и $b(x, y, t)$ соответствующие плотности распределния.

Пусть $\lambda=\lambda(x, t)$ - решение уравнения

$$
\lambda(x, t)=x+\int_{0}^{t} c(\lambda(x, s)) d s .
$$

По определению процесса $X(x, t)$ имеем [1]

$$
\begin{aligned}
F(x, y, t+\Delta t)= & (1-\mu \Delta t) F(x, \lambda(y,-\Delta t), t)+ \\
& +\mu \Delta t B(x, \lambda(y, \Delta t), t)+o(\Delta t), \\
B(x, y, t+\Delta t)= & (1-\mu \Delta t) B(x, \lambda(y, \Delta t), t)+ \\
& +\mu \Delta t F(x, \lambda(y,-\Delta t), t)+o(\Delta t), \Delta t \rightarrow 0 .
\end{aligned}
$$

Из (3.6), (3.7) вытекают дифференциальные уравнения

$$
\begin{aligned}
& \frac{\partial F}{\partial t}=-c(y) \frac{\partial F(x, y, t)}{\partial y}+\mu(B(x, y, t)-F(x, y, t)), \\
& \frac{\partial B}{\partial t}=c(y) \frac{\partial B(x, y, t)}{\partial y}+\mu(F(x, y, t)-B(x, y, t)) .
\end{aligned}
$$

После дифференцирования этих уравнений по $y$ имеем

$$
\begin{aligned}
& \frac{\partial f}{\partial t}=-\frac{\partial c(y) f(x, y, t)}{\partial y}+\mu(b(x, y, t)-f(x, y, t)), \\
& \frac{\partial b}{\partial t}=\frac{\partial c(y) b(x, y, t)}{\partial y}+\mu(f(x, y, t)-b(x, y, t)) .
\end{aligned}
$$

Заметим, что $p(x, y, t)=f(x, y, t)+b(x, y, t)$. Обозначим $w(x, y, t)=f(x, y, t)-$ $b(x, y, t)$. Тогда получим

$$
\begin{gathered}
\frac{\partial p}{\partial t}=-\frac{\partial c(y) w(x, y, t)}{\partial y} \\
\frac{\partial w}{\partial t}=-\frac{\partial c(y) p(x, y, t)}{\partial y}-2 \mu w(x, y, t),
\end{gathered}
$$

отсюда вытекает (3.1). Начальные условия (3.2) совпадают с (2.13).

ЗАмЕчАниЕ 3.1. Уравнение (3.1) назовем прямым уравнением Колмогорова (или уравнением Фоккера-Планка). Отметим, что оператор в правой части (3.1) формально сопряжен оператору в $(2.3)$.

ЗАмЕчАнИЕ 3.2. Имеются следуюшие очевидные соотношения между $f, b$ и $p^{ \pm}$ (см. (2.12)):

$$
\begin{aligned}
\frac{1}{2} p^{+}(x, y, t) & =f(y, x, t), & \frac{1}{2} p^{-}(x, y, t) & =b(y, x, t), \\
p^{+}(x, y, t) & =p^{-}(y, x, t), & p(x, y, t) & =p(y, x, t) .
\end{aligned}
$$




\section{4. ТЕЛЕГРАФНЫЕ ПРОЦЕССЫ С БАРЬЕРАМИ}

4.1. Отражающие барьеры. Предположим, что частица, блуждаюшая по закону (1.1), изменяет направление скорости на противоположное в точке $y=a>x$. Иными словами, рассмотрим процесс

$$
X^{\mathrm{ref}}(x, t)= \begin{cases}X(x, t), & t \leq \tau=\tau^{1} \\ a-(-1)^{N\left(\tau^{k}\right)} \int_{\tau^{k}}^{t}(-1)^{N(s)} c\left(X^{\mathrm{ref}}(x, s)\right) d s, & \tau^{k}<t \leq \tau^{k+1} \\ k=1,2, \ldots & \end{cases}
$$

Здесь через $\tau^{k}=\tau^{k}(x, a), k=1,2, \ldots$, обозначен момент $k$-го отражения:

$$
\tau=\tau^{1}=\inf \left\{t>0: X^{\mathrm{ref}}(x, t)=a\right\}
$$

- момент первого достижения частицей точки $y=a$;

$$
\tau^{k}=\inf \left\{t>\tau^{k-1}: X^{\mathrm{ref}}(x, t)=a\right\}, \quad k \geq 2
$$

Отметим, что после каждого столкновения скорость частицы $c(a)$ отрицательна независимо от знака начальной скорости. Кроме того, после каждого столкновения с барьером в точке $y=a$ процесс начинается заново. В самом деле, при $t \in\left(\tau^{k}, \tau^{k+1}\right]$ имеем

$$
\begin{aligned}
X^{\mathrm{ref}}(x, t) & =a-(-1)^{N\left(\tau^{k}\right)} \int_{\tau^{k}}^{t}(-1)^{N(s)} c\left(X^{\mathrm{ref}}(x, s)\right) d s= \\
& =a-\int_{0}^{t-\tau^{k}}(-1)^{N\left(s+\tau^{k}\right)-N\left(\tau^{k}\right)} c\left(X^{\mathrm{ref}}\left(x, s+\tau^{k}\right)\right) d s= \\
& =a-\int_{0}^{t-\tau^{k}}(-1)^{N^{\prime}(s)} c\left(X^{\mathrm{ref}}\left(x, s+\tau^{k}\right)\right) d s .
\end{aligned}
$$

Здесь $N^{\prime}(s)=N\left(s+\tau^{k}\right)-N\left(\tau^{k}\right)$ - число пуассоновских событий, произошедших после момента $\tau^{k}$, имеет то же распределение, что и $N(s)$. Мы будем предполагать в этом разделе, что $c=c(x), x<a,-$ функция класса $C^{1}$. Пусть $\varphi=\varphi(x), x<a,-$ некоторая гладкая функция, причем $\left.\varphi^{\prime}\right|_{x=a-0}=0$. Рассмотрим функцию $u^{\mathrm{ref}}(x, t)=$ $E \varphi\left(X^{\mathrm{ref}}(x, t)\right)$.

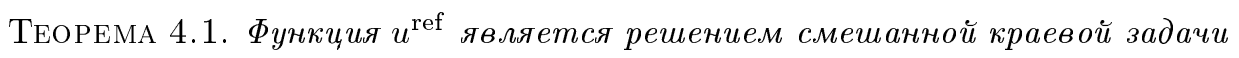

$$
\begin{gathered}
\frac{\partial^{2} u(x, t)}{\partial t^{2}}+2 \mu \frac{\partial u(x, t)}{\partial t}=c(x) \frac{\partial}{\partial x} c(x) \frac{\partial}{\partial x} u(x, t), \quad x<a, \quad t>0, \\
\left.u\right|_{t=0}=\varphi(x),\left.\quad u_{t}\right|_{t=0}=0, \quad x<a, \\
\left.u_{x}\right|_{x=a-0}=0, \quad t>0 .
\end{gathered}
$$


ДокАЗАТЕЛЬСТво. Пусть $\bar{c}=\bar{c}(x), x \in(-\infty, \infty)$,- четное продолжение функции $c(x)$, первоначально заданной при $x<a$. Обозначим через $\bar{X}(x, t), x \in(-\infty, \infty), t>0$, процесс вида (1.1), отвечающий полю скоростей $\bar{c}(x)$.

Ключевую роль в доказательстве теоремы 4.1 играет следуюшая лемма.

Лемма 4.1. При $\tau^{2 k-1} \leq t<\tau^{2 k}$ распределения $\bar{X}(x, t)$ u $X^{+}\left(a, t-\tau^{2 k-1}\right)$ совпадают, а при $\tau^{2 k} \leq t<\tau^{2 k+1}$ совпадают распределения $\bar{X}(x, t)$ и $X^{-}\left(a, t-\tau^{2 k}\right)$, $k \in \mathbf{N}$.

Здесь процессы $X^{+}$и $X^{-}$определены равенствами $(2.1),(2.2)$, моменты $\tau^{k}$ заданы в (4.1), (4.2).

ДокАЗАТЕльСТво леммы. Приведем доказательство для $\tau=\tau^{1}<t \leq \tau^{2}$ (в общем случае рассуждения аналогичны). Заметим, что из определения $\tau^{1}(4.1)$ имеем

$$
\begin{aligned}
\bar{X}(x, t) & =a+\xi \int_{\tau}^{t}(-1)^{N(s)} \bar{c}(\bar{X}(x, s)) d s= \\
& =a+\xi(-1)^{N(\tau)} \int_{0}^{t-\tau}(-1)^{N^{\prime}(s)} \bar{c}(\bar{X}(x, s+\tau)) d s
\end{aligned}
$$

Для завершения доказательства осталось заметить, что $\xi(-1)^{N(\tau)}=1$, т.к. в случае $\xi=+1$ число пуассоновских событий до момента $\tau$ четно, тогда как при $\xi=-1$ это число нечетно. Лемма 4.1 доказана.

Перейдем к докАЗАТЕльствУ теоремы 4.1. Отметим, что

$$
u^{\mathrm{ref}}(x, t)=E \varphi\left(X^{\mathrm{ref}}(x, t)\right)=E\left[\varphi\left(X^{\mathrm{ref}}(x, t)\right) I_{\left\{t<\tau^{1}\right\}}+\varphi\left(X^{\mathrm{ref}}(x, t)\right) I_{\left\{t \geq \tau^{1}\right\}}\right] .
$$

Обозначим через $\bar{\varphi}=\bar{\varphi}(x)$ четное продолжение функции $\varphi$ вправо от точки $y=a$. При $t<\tau^{1}$ имеем $X^{\mathrm{ref}}(x, t)=X(x, t)$, а в силу (4.3) на каждом из интервалов $\tau^{k} \leq t<\tau^{k+1}$, $k \in \mathbf{N}, X^{\mathrm{ref}}(x, t)=X^{-}\left(a, t-\tau^{k}\right)$. Отсюда и из леммы 4.1 в силу четности продолжения функции $\varphi$ вытекает, что

$$
u^{\mathrm{ref}}(x, t)=E\left[\bar{\varphi}(\bar{X}(x, t)) I_{\left\{t<\tau^{1}\right\}}+\bar{\varphi}(\bar{X}(x, t)) I_{\left\{t \geq \tau^{1}\right\}}\right]=E \bar{\varphi}(\bar{X}(x, t)) .
$$

Правая часть этого равенства $E \bar{\varphi}(\bar{X}(x, t)) \equiv u(x, t)$ в силу теоремы 2.2 является решением задачи Коши (2.3), (2.9). Поскольку $\bar{c}$ и $\bar{\varphi}$ - четные относительно $a$ функции, то задачи $(2.3),(2.9)$ и (4.4)-(4.6) эквивалентны.

4.2. Поглощающие барьеры. Предположим теперь, что частица поглощается в точке $y=a>x$. Это означает, что процесс (обозначим его $X^{a b s}(x, t), x \in(-\infty, a]$, $t>0)$ при $t<\tau^{1}$ совпадает с $X(x, t)$, а при $t \geq \tau^{1}$ имеем $X^{a b s}(x, t)=a$. Пусть $u^{\mathrm{abs}}(x, t)=E \varphi\left(X^{\mathrm{abs}}(x, t)\right)$. Рассмотрим также функции распределения $F^{\mathrm{abs}}(x, y, t)$ и $B^{\text {abs }}(x, y, t)$, определенные для процесса $X^{\text {abs }}$, так же как в (3.3), (3.4). Пусть $f^{\text {abs }}$ и $b^{\mathrm{abs}}$ - соответствуюшие плотности. Очевидно, что $p^{\mathrm{abs}}=f^{\mathrm{abs}}+b^{\mathrm{abs}}$, где $p^{\mathrm{abs}}-$ плотность для $X^{\text {abs }}$.

Аналогично теореме 3.1 устанавливается следующее предложение. 
ПРЕДЛОЖЕНИЕ: 1) $f^{\text {abs }}$ является решением задачи

$$
\begin{gathered}
\frac{\partial^{2}}{\partial t^{2}} u+2 \mu \frac{\partial}{\partial t} u=\frac{\partial}{\partial y} c(y) \frac{\partial}{\partial y} c(y) u, \quad y<a \\
\left.u\right|_{t=0}=\frac{1}{2} \delta(x-y),\left.\quad u_{t}\right|_{t=0}=-\frac{1}{2} \frac{\partial}{\partial y} c(y) \delta(x-y), \quad x, y<a \\
\left.\left(\frac{\partial u}{\partial t}+c(y) \frac{\partial u}{\partial y}+\mu u\right)\right|_{y=a-0}=0
\end{gathered}
$$

2) $b^{\mathrm{abs}}$ является решением задачи

$$
\begin{gathered}
\frac{\partial^{2}}{\partial t^{2}} u+2 \mu \frac{\partial}{\partial t} u=\frac{\partial}{\partial y} c(y) \frac{\partial}{\partial y} c(y) u, \quad y<a \\
\left.u\right|_{t=0}=\frac{1}{2} \delta(x-y),\left.\quad u_{t}\right|_{t=0}=\frac{1}{2} \frac{\partial}{\partial y} c(y) \delta(x-y), \quad x, y<a \\
\left.u\right|_{y=a-0}=0 .
\end{gathered}
$$

Справедлива, следовательно, следующая теорема.

ТеОрема 4.2. Пусть $\varphi \in C(-\infty, a], \operatorname{mozдa~}$

$$
u^{\mathrm{abs}}=\left\langle f^{\mathrm{abs}}(x, \cdot, t), \varphi\right\rangle+\left\langle b^{\mathrm{abs}}(x, \cdot, t), \varphi\right\rangle,
$$

где $f^{\mathrm{abs}}$ и $b^{\mathrm{abs}}-$ решения задач (4.7)-(4.9) и $\left(4.7^{\prime}\right)-\left(4.9^{\prime}\right)$, соответственно.

\section{5. РЕШЕНИЯ ТЕЛЕГРАФНОГО УРАВНЕНИЯ}

В этом разделе приводятся формулы для решения задач (2.3), (2.9), (4.4)-(4.6), (4.7)-(4.9), (4.7')-(4.9'). Построим сначала решение задачи Коши (2.3), (2.9). Для этого наряду с телеграфным уравнением (2.3) рассмотрим следующее уравнение:

$$
\frac{\partial^{2} v}{\partial t^{2}}=c(x) \frac{\partial}{\partial x} c(x) \frac{\partial v}{\partial x}+\mu^{2} v
$$

При $\mu^{2}<0$ это уравнение известно как уравнение Клейна-Гордона.

Введем бихарактеристики $\lambda^{ \pm}$, обшие для уравнений $(2.3),(3.1)$ и (5.1). Пусть $\lambda^{+}=\lambda(x, t)$ и $\lambda^{-}=\lambda(x,-t), t \in(-\infty, \infty)$, где функция $\lambda(x, t)$ определена в $(3.5)$. Заметим, что $\lambda^{ \pm}-$решения следующих уравнений:

$$
\begin{aligned}
& \lambda^{+}(x, t)=x+\int_{0}^{t} c\left(\lambda^{+}(x, s)\right) d s, \\
& \lambda^{-}(x, t)=x-\int_{0}^{t} c\left(\lambda^{-}(x, s)\right) d s .
\end{aligned}
$$


Теорема 5.1. Решение задачи (2.3), (2.4) имеет вид

$$
\begin{aligned}
u(x, t)=\frac{1}{2} e^{-\mu t}\left[\varphi\left(\lambda^{+}(x, t)\right)+\varphi\left(\lambda^{-}(x, t)\right)+\right. & \\
& \left.\quad+\int_{-t}^{t} \varphi(\lambda(x, s))\left(\mu I_{0}\left(\mu \sqrt{t^{2}-s^{2}}\right)+\frac{\partial}{\partial t} I_{0}\left(\mu \sqrt{t^{2}-s^{2}}\right)\right) d s\right]
\end{aligned}
$$

здесь

$$
I_{0}(x)=\sum_{n=0}^{\infty} \frac{x^{2 n}}{2^{2 n}(n !)^{2}}
$$

- модифицированная функиия Бесселя.

ЗАмЕчАнИЕ 5.1. После замены переменных $\lambda(x, s)=y$ в интеграле формула (5.4) принимает вид

$$
\begin{aligned}
u(x, t)= & \frac{1}{2} e^{-\mu t}\left[\varphi\left(\lambda^{+}(x, t)\right)+\varphi\left(\lambda^{-}(x, t)\right)+\right. \\
& \left.+\int_{\lambda^{-}(x, t)}^{\lambda^{+}(x, t)} \frac{\varphi(y)}{c(y)}\left(\mu I_{0}\left(\mu \sqrt{t^{2}-\sigma(y, x)^{2}}\right)+\frac{\partial}{\partial t} I_{0}\left(\mu \sqrt{t^{2}-\sigma(y, x)^{2}}\right)\right) d y\right]
\end{aligned}
$$

здесь $\sigma=\sigma(y, x)$ - решение уравнения $\lambda(x, \sigma)=y$.

ДоКАЗАТЕЛЬСТВо теоремы 5.1 основано на следующих леммах.

Лемма 5.1. Функиия $u=u(x, t)$ - решение задачи (2.3), (2.9) тогда и только тогда, когда $u=e^{-\mu t} v$, где $v=v(x, t)$ - решение уравнения (5.1) с начальньми условиями

$$
\left.v\right|_{t=0}=\varphi,\left.\quad v_{t}\right|_{t=0}=\mu \varphi
$$

Обозначим

$$
\begin{aligned}
Z(x, t ; \psi) & =\frac{1}{2} \int_{0}^{t}\left(\psi\left(\lambda^{+}(x, s)\right)+\psi\left(\lambda^{-}(x, s)\right)\right) I_{0}\left(\mu \sqrt{t^{2}-s^{2}}\right) d s \equiv \\
& \equiv \frac{1}{2} \int_{-t}^{t} \psi(\lambda(x, s)) I_{0}\left(\mu \sqrt{t^{2}-s^{2}}\right) d s .
\end{aligned}
$$

Лемма 5.2. Решение $v(x, t)$ уравнения (5.1) с начальными условиями

$$
\left.u\right|_{t=0}=\varphi,\left.\quad u_{t}\right|_{t=0}=\psi
$$

имеет вид

$$
v(x, t)=Z(x, t ; \psi)+Z_{t}(x, t ; \varphi) .
$$

Для доказательства теоремы 5.1 достаточно подставить $\mu \varphi$ вместо $\psi$ в (5.6) и выполнить дифференцирование по $t$. Доказательства лемм 5.1 и 5.2 состоят из прямых и несложных вычислений и здесь опушены.

Из теоремы 5.1 вытекают явные формулы как для распределения $p=p(x, y, t)$ процесса $X(x, t)$ ("свободное" движение), так и для $p^{\mathrm{ref}}(x, y, t)$ и $p^{\mathrm{abs}}(x, y, t)$ (в случае движений с отражаюшим и поглощаюшим барьерами). Из следствия 2.1 и теоремы 5.1 вытекает следуюшая формула для распределения процесса $X(x, t)$. 
СлеДСТВИЕ 5.1. Плотность распределения $p(x, y, t)$ процесса $X(x, t)$ имеет вид (ср. с формулой (2.5) в [2])

$$
\begin{aligned}
& p(x, y, t)=\frac{1}{2} e^{-\mu t}\left[\delta\left(y-\lambda^{+}(x, t)\right)+\delta\left(y-\lambda^{-}(x, t)\right)+\right. \\
& \left.+\frac{1}{c(y)}\left(\mu I_{0}\left(\mu \sqrt{t^{2}-\sigma(y, x)^{2}}\right)+\frac{\partial}{\partial t} I_{0}\left(\mu \sqrt{t^{2}-\sigma(y, x)^{2}}\right)\right) \chi_{\left[\lambda^{-}(x, t), \lambda^{+}(x, t)\right]}(y)\right] .
\end{aligned}
$$

Здесь $\chi_{\Delta}(y)=1$, если $y \in \Delta, u \chi_{\Delta}(y)=0$, если $y \notin \Delta$.

ЗАмЕчАнИЕ 5.2. Из доказанных выше утверждений ясно, что решение задач (2.3), $(2.9)$ и $(3.1),(3.2)$ сушествует при $t \in[0,+\infty)$, если бихарактеристики $\lambda^{+}$и $\lambda^{-}$уходят на бесконечность за бесконечное время. Чтобы гарантировать это, достаточно потребовать выполнения условий

$$
\begin{gathered}
c(x) \geq c_{0}>0, \quad x \in(-\infty, \infty) \\
\int_{0}^{+\infty} \frac{d y}{c(y)}=\infty, \quad \int_{-\infty}^{0} \frac{d y}{c(y)}=\infty
\end{gathered}
$$

На основе формулы (5.4) из теоремы 2.1 и лемм 5.1 и 5.2 получаются формулы для плотностей рапределения $p^{+}(x, y, t), p^{-}(x, y, t), p^{\mathrm{ref}}(x, y, t)$ и $p^{\mathrm{abs}}(x, y, t)$.

СлеДСтвИЕ 5.2. Плотности распределения $p^{ \pm}(x, y, t)$ процессов $X^{ \pm}$имеют вид (ср. с формулой (2.17) в [2])

$$
p^{ \pm}(x, y, t)=p(x, y, t) \mp \frac{e^{-\mu t}}{2} \chi_{\left[\lambda^{-}(x, t), \lambda^{+}(x, t)\right]}(y) \frac{\partial}{\partial y} I_{0}\left(\mu \sqrt{t^{2}-\sigma(y, x)^{2}}\right)
$$

Явные формулы для $p^{ \pm}(x, y, t)$ можно получить, используя (5.7).

Чтобы выписать формулы для $p^{\mathrm{ref}}(x, y, t)$ и $p^{\mathrm{abs}}(x, y, t)$, рассмотрим снова процесс $\bar{X}(x, t)$, порожденный четным относительно точки $x=a$ полем скоростей $\bar{c}(x)$. Всюду ниже $\bar{p}=\bar{p}(x, y, t)$ - соответствуюшая этому процессу плотность распределения.

СледСТВИЕ 5.3. Плотность распределения $p^{\mathrm{ref}}(x, y, t)$ прочесса с отражсением $X^{\mathrm{ref}}(x, t)$ имеет вид

$$
p^{\mathrm{ref}}(x, y, t)=\bar{p}(x, y, t)+\bar{p}(2 a-x, y, t), \quad x, y<a
$$

ДокАЗАТЕльство. Как видно из теоремы $4.1, p^{\text {ref }}(x, y, t)$ удовлетворяет задаче $(2.3),(2.9)$ с $c(x)=\bar{c}(x), \varphi(x)=\delta(x-y)+\delta(2 a-x-y)$. Отсюда и из следствия 2.1 вытекает равенство (5.11).

Явные формулы для $p^{\text {ref }}$ нетрудно получить из (5.7). 
СлЕДСтвиЕ 5.4. Плотность распределения $p^{\mathrm{abs}}(x, y, t)$ прочесса с поглощением $X^{\text {abs }}$ имеет вид (ср. с формулами (3.3), (3.4) в [2])

$$
p^{\text {abs }}(x, y, t)=\bar{p}(x, y, t)-\bar{p}^{-}(x, 2 a-y, t), \quad x, y<a .
$$

Явные формулы для $p^{\mathrm{abs}}$ вытекают из (5.7) и (5.10).

ДокАЗАТЕЛЬСТво. Заметим сначала, что

$$
b^{\mathrm{abs}}(x, y, t)=\frac{1}{2}\left(\bar{p}^{-}(x, y, t)-\bar{p}^{-}(x, 2 a-y, t)\right), \quad x, y<a,
$$

- решение задачи $\left(4.7^{\prime}\right)-\left(4.9^{\prime}\right)$, а

$$
f^{\text {abs }}(x, y, t)=\frac{1}{2}\left(\bar{p}^{+}(x, y, t)-\bar{p}^{-}(x, 2 a-y, t)\right), \quad x, y<a,
$$

- решение задачи (4.7)-(4.9).

Эти утверждения проверяются непосредственной подстановкой $(5.13)$ в $\left(4.7^{\prime}\right)-\left(4.9^{\prime}\right)$ и (5.14) в (4.7)-(4.9). При этом используются следствие 2.1, соотношения $(3.8)$ и тождество

$$
-\frac{\partial}{\partial y} c(y) \delta(x-y)=c(x) \delta^{\prime}(x-y) .
$$

Складывая (5.13) и (5.14), получаем (5.12).

Работа поддержана Российским фондом фундаментальных исследований (код проекта: 96-01-01169).

\section{Список литературы}

[1] E. Orsingher. Stoch. Proc. Appl. 1990. V. 38. P. 49-66.

[2] E. Orsingher. Random Oper. and Stoch. Equations. 1995. V. 3. № 1. P. 9-21.

[3] S. K. Foong. Phys. Rev. A. 1992. V. 46. P. 707-710.

[4] J.Masoliver, J. M. Porra, G. H. Weiss. Phys. Rev. A. 1992. V. 45. P. 2222-2227.

[5] В. Кляцкин. Стохастические уравнения и волны в случайных неоднородных средах. М.: Наука, 1980.

[6] S. Goldstein. Quart. J. Mech. Appl. Math. 1951. V. 4. P. 129-156.

[7] М. Кач. Вероятность и смежные вопросы физики. М.: Наука, 1965.

[8] E. Orsingher. Stoch. Proc. Appl. 1985. V. 21. P. 129-156.

[9] Ю. М. Кабанов. Теория вероятн. и ее примен. 1992. Т. 37. В. 2. С. 425-426.

[10] А. И. Комеч. Линейные уравнения в частных производных с постоянными коэффициентами. В кн.: Итоги науки и техники. Соврем. проблемы математики. Фундаментальные направления. Т. 31. М.: ВИНИТИ, 1988. С. 127-261.

Поступила в редакцию 20.XI.1996 г., после доработки 27.II.1997 г.

\section{N. E. Ratanov}

\section{RANDOM MOTION OF PARTICLE IN INHOMOGENEOUS 1D MEDIA WITH REFLECTING AND ABSORBING BARRIERS}

A random motion in 1D inhomogeneous media is considered. A moving particle changes abruptly a direction of velocity at Poisson times. The backward and forward Kolmogorov's equations describing this motion are derived. The explicit formulas for the probability distributions are obtained both for this motion and for the similar motions with reflecting and absorbing barriers. 